\title{
Mechanical Properties of MJ-class Toroidal Magnet Wound by Composite HTS Conductor
}

\author{
Ming Qiu, Shuangquan Rao, Jiahui Zhu, PanPan Chen, Shanshan Fu, Weijia Yuan, Jun Gong
}

\begin{abstract}
An MJ-class superconducting magnetic energy storage (SMES) system has a wide range of potential applications in electric power systems. The composite HTS conductor, which has the advantages of carrying large critical currents and withstanding high magnetic fields, is suitable for winding an MJclass magnet coil. However, the Lorentz force of an HTS wire is so large that its induced mechanical stresses should be examined to ensure that the magnet is in good condition. By means of the equivalent material properties method and the sequential coupling method, this paper studies the mechanical properties of a 3 MJ toroidal SMES magnet wound by a composite HTS conductor. Based on the electromagnetic-structural coupling analysis, the Von-Mises stress, the radial stress, and the hoop stress of a magnet coil are calculated and employed to validate the stability of the MJ-class toroidal SMES magnet.
\end{abstract}

Index Terms-SMES, composite HTS conductor, equivalent material properties, sequential coupling, mechanical analysis

\section{INTRODUCTION}

Superconducting Magnetic Energy Storage (SMES) $N_{\text {systems have the advantages of high power density and a }}$ fast response speed. They can be used to compensate voltage sags and mitigate power fluctuations in an electrical grid [1]. SMES technology can also be used to facilitate the gridconnection of renewable energy, increase the stability of a power grid and the quality of power supply [2]. In the near future, an MJ-class SMES system is expected to play an important role in power grids. An MJ-class SMES system carries large currents and creates a high field in operation. An SMES magnet is considered to be a key component of the SEMS system. If an SMES magnet were wound by a commercial superconductor, such as a YBCO tape with the cross-section of $4.4 \mathrm{~mm} \times 0.1 \mathrm{~mm}$, whose critical current is limited to 100 A 300 A (@77 K, self-field), it would be very difficult to achieve large-capacity energy storage higher than the MJ-class considering the expensive cooling cost. Consequently, it would not provide a favorable condition for the applications of MJ-class SMES systems in power grids.

Compared with a parallel stacked wire, a composite HTS

This work was supported by the China State Grid Corporation science and technology project under Grant: DG71-14-034; DG71-14-045.

Ming Qiu, Shuangquan Rao, Jiahui Zhu, PanPan Chen and Shanshan Fu are with the China Electric Power Research Institute, Beijing, China (e-mail: qiuming@epri.sgcc.com.cn;591854336@qq.com;zhujiahui@epri.sgcc.com.cn; chenpanpan@epri.sgcc.com.cn; shanshanfu2012@163.com).

Weijia Yuan is with University of Bath, Bath, BA2 7AY, United Kingdom (e-mail: W.Yuan@bath.ac.uk).

Jun Gong is with the Beijing Jiaotong University, Beijing, China (e-mail: 15121407@bjtu.edu.cn). (Corresponding author: Ming Qiu.) conductor has the ability to both carry large currents and withstand high magnetic fields, which makes it a good choice for winding magnet coils in high fields [3]-[5]. Additionally, in order to increase storage energy and reduce electromagnetic pollution, the toroid-type SMES magnet is desirable for an MJ-class SMES system.

On the other hand, toroid-type SMES magnet coils wound by composite HTS conductors may suffer from a great Lorentz force due to the interaction between large currents and high fields. Considerable mechanical stresses caused by large Lorentz force will influence the instability of the magnet coil and even damage the HTS wire. Moreover, HTS wires are ceramic and brittle materials, which are easily damaged by large stresses. Therefore, an analysis of the mechanical properties of a magnet coil wound by a composite HTS conductor is essential to ensuring the stability of an SMES magnet during operation.

In this paper, a $3 \mathrm{MJ}$ toroidal magnet wound by composite HTS conductors is examined for its mechanical properties. Firstly, the magnetic field and Lorentz force of a $3 \mathrm{MJ}$ toroidal SMES magnet are calculated using COMSOL software based on the finite element method (FEM). Secondly, the composite HTS conductor is simplified as an equivalent orthotropic material using the equivalent material properties method. Thirdly, the sequential coupling analysis method is used to calculate the mechanical stresses of a $3 \mathrm{MJ}$ toroidal magnet coil, and the results are discussed in detail. These fundamental data will be effectively used to investigate the mechanical properties of an MJ-class toroidal magnet wound by a composite HTS conductor.

\section{COMPOSITE HTS CONDUCTORS}

In this paper, a type of composite HTS conductor is used to wind a toroidal SMES magnet, whose critical current is as high as $1 \mathrm{kA}$ at $77 \mathrm{~K}$ (self-field). As shown in Fig. 1, the composite HTS conductor mainly includes: (1) A 4-ply REBCO coated conductor with its major features shown in Table I (Note: The 4-ply REBCO coated conductor is arrayed in a twisted and stacked manner.); (2) An aluminum alloy inner layer; (3) An aluminum alloy outer layer; (4) A cooling channel.

The above-mentioned composite HTS conductor is used to wind an MJ-class toroidal SMES magnet. The conductor has a cooling medium channel, so a forced flow cooling method is used for an MJ-class toroidal SMES magnet. A circulation cooling system is formed when the cooling medium (liquid helium in this paper) continuously flows through the cooling 
channel, which has high cooling efficiency.

TABLE I

SPECIFICATIONS OF THE REBCO COATED CONDUCTOR

\begin{tabular}{llll}
\hline \hline Width & Thickness & Critical current & Critical tensile strength \\
\hline $5.5 \mathrm{~mm}$ & $0.14 \mathrm{~mm}$ & $280 \mathrm{~A}(@ 77 \mathrm{~K}, \mathrm{SF})$ & $>150 \mathrm{MPa}$ \\
\hline \hline
\end{tabular}

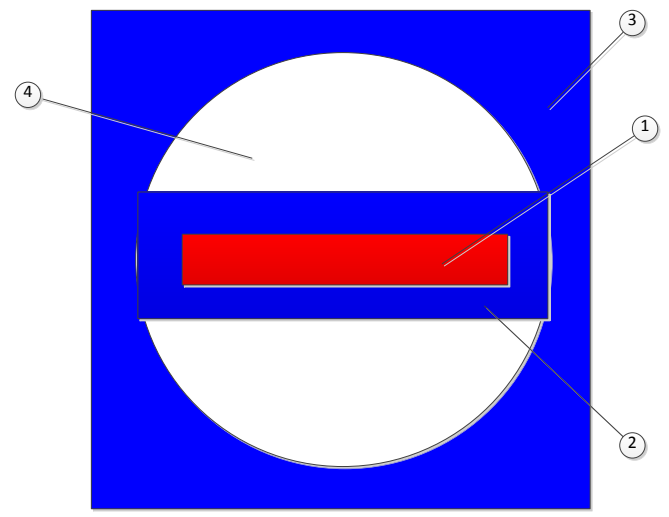

Fig. 1. Schematic diagram of composite HTS conductor

In addition, there are two advantages when an MJ-class toroidal SMES magnet is wound by a composite HTS conductor: 1) The 4-ply REBCO coated conductor is arrayed in a twisted and stacked way, which can reduce AC losses during charge and discharge and improve the thermal stability of the composite HTS conductor. 2) The anisotropy of HTS wires can be reduced to make the distribution of currents more uniform, which helps to increase the critical current of an SMES magnet.

\section{EQUivalENT MATERIAL PROPERTIES CALCULATION}

To accurately reflect the mechanical properties of magnet coil, equivalent material property method is used in this paper. Therefore, a composite HTS conductor can be simplified as an equivalent orthotropic material to represent the mechanical properties of magnet coil.

In addition, compared with the composite HTS conductor size, the thickness of REBCO coated conductor is quite thin, and its mechanical strength is much weaker than aluminum alloy. The composite HTS conductor model is simplified as a combination of an aluminum alloy clad layer and an insulation layer, shown in Fig. 2.

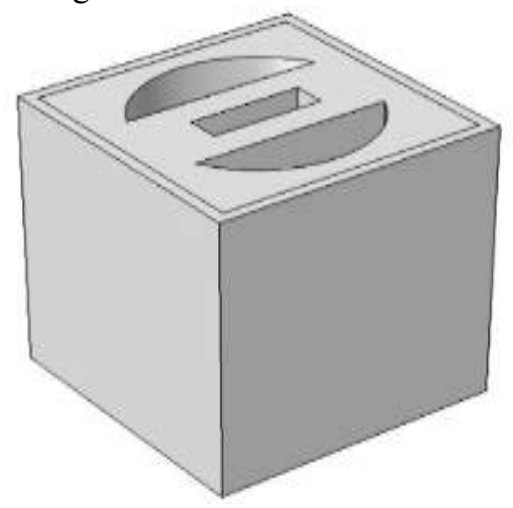

Fig. 2. Simplified model of composite HTS conductor

\section{A. Equivalent Young's Modulus and Poisson's Ratio}

An orthotropic material's stress-strain relationship can be described by the generalized Hooke's law [6].

$$
\left\{\begin{array}{l}
\varepsilon_{x}=\frac{\sigma_{x}}{E_{x}}-\frac{v_{x y} \sigma_{y}}{E_{x}}-\frac{v_{x z} \sigma_{z}}{E_{x}}, \gamma_{x y}=\frac{\tau_{x y}}{G_{x y}} \\
\varepsilon_{y}=\frac{\sigma_{y}}{E_{y}}-\frac{v_{y x} \sigma_{x}}{E_{y}}-\frac{v_{y z} \sigma_{z}}{E_{y}}, \gamma_{y z}=\frac{\tau_{y z}}{G_{y z}} \\
\varepsilon_{z}=\frac{\sigma_{z}}{E_{z}}-\frac{v_{z x} \sigma_{x}}{E_{z}}-\frac{v_{z y} \sigma_{y}}{E_{z}}, \gamma_{z x}=\frac{\tau_{z x}}{G_{z x}}
\end{array}\right.
$$

Where, $\sigma, \varepsilon(i=x, y, z)$ are normal stress and normal stain in directions $x, y, z$, respectively. $\gamma, \tau(i, j=x, y, z)$ are shear stress and shear stain respectively. $E_{i}(i=x, y, z)$ are Young modulus. $v_{i j}(i, j=x, y, z)$ are Poisson's ratio, and $G_{i j}$ are shear modulus. The symmetry conditions $v_{i j} / E_{i}=v_{j i} / E_{j}(i, j=x, y$, $z$ ) must be satisfied.

Taking $E_{x}$ and $v_{x y}$ as an example, $E_{y}, E_{z}$ and $v_{y z}, v_{x z}$ can be calculated with the same approach. When imposing a constant X-direction of pressure $P_{x}$ on the composite HTS conductor, (2) can be obtained to calculate $E_{x}$ and $v_{x y}$.

$$
\left\{\begin{array}{c}
\varepsilon_{x}=\frac{U X_{a v}}{L_{x}}, \varepsilon_{y}=\frac{U Y_{a v}}{L_{y}}, \varepsilon_{x}=\frac{U Z_{a v}}{L_{z}} \\
\sigma_{x}=-P_{x}, \sigma_{y}=0, \sigma_{z}=0 \\
E_{x}=\frac{\sigma_{x}}{\varepsilon_{x}}, v_{x y}=-\frac{\varepsilon_{y}}{\varepsilon_{x}}, v_{x z}=-\frac{\varepsilon_{z}}{\varepsilon_{x}}
\end{array}\right.
$$

Where, $U X_{a v}, U Y_{a v}, U Z_{a v}$ are average displacements in directions $x, y, z . L_{x}, L_{y}, L_{z}$ are the lengths of the composite HTS conductor in directions $x, y, z$. The X-component of the displacement pattern of an equivalent material model when calculating $E_{x}$ and $v_{x y}$ is shown in Fig. 3.

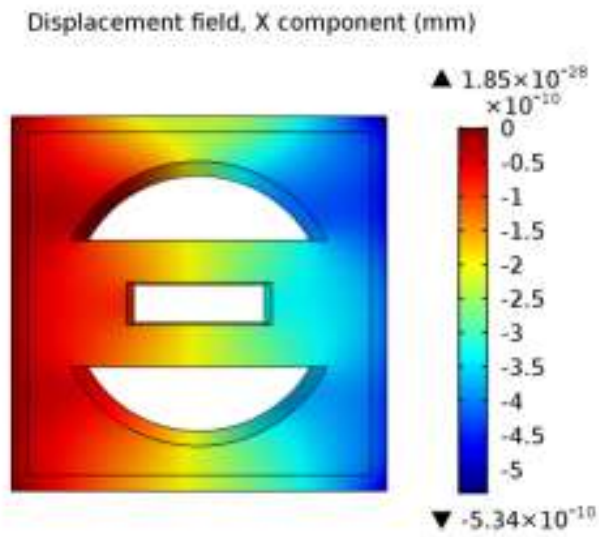

Fig. 3. X-component of the displacement pattern of an equivalent material model when calculating $E_{x}$ and $v_{x y}$

\section{B. Equivalent Shear Modulus}

Similarly, taking $G_{x y}$ as an example, $G_{y z}, G_{x z}$ can be calculated with the same approach. When imposing a constant X-direction of force $F_{x}$ on the composite HTS conductor, (3) can be obtained to calculate $G_{x y}$. 


$$
\left\{\begin{array}{l}
\tau_{x y}=\frac{\sum F_{x}}{A_{y z}}, \gamma_{x y}=\frac{U X_{a v}}{L_{y}}, G_{x y}=\frac{\tau_{x y}}{\gamma_{x y}} \\
\tau_{y z}=\frac{\sum F_{y}}{A_{x z}}, \gamma_{y z}=\frac{U Y_{a v}}{L_{z}}, G_{y z}=\frac{\tau_{y z}}{\gamma_{y z}} \\
\tau_{x z}=\frac{\sum F_{z}}{A_{x y}}, \gamma_{x z}=\frac{U X_{a v}}{L_{z}}, G_{x z}=\frac{\tau_{x z}}{\gamma_{x z}}
\end{array}\right.
$$

Where, $\sum F_{i}(i=x, y, z)$ are the total nodal force in directions $x, y, z$. Fig. 4 shows the X-component of the displacement pattern of an equivalent material model when calculating $G_{x y}$.

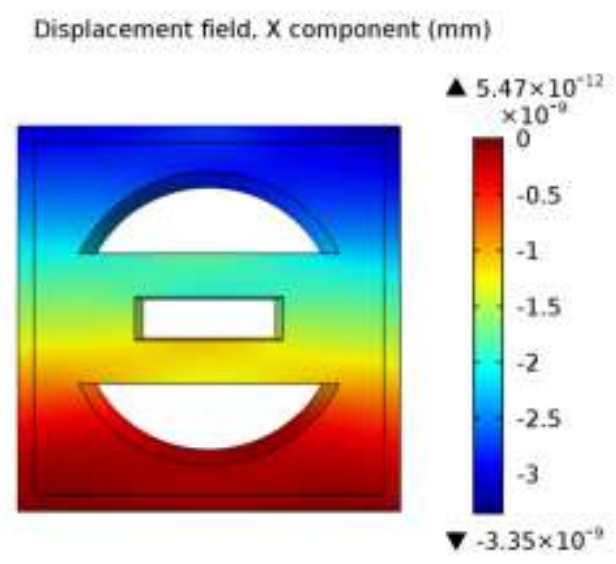

Fig. 4. X-component of the displacement pattern of an equivalent material model when calculating $G_{x y}$

Based on the equivalent material properties method, the equivalent orthotropic material properties of magnet coil are listed in TABLE II.

TABLE II

THE Equivalent Material Properties of Magnet CoIL

\begin{tabular}{llll}
\hline \hline Direction & Young modulus & Poisson's ratio & Shear modulus \\
\hline $\mathrm{x}(\mathrm{xy})$ & $32.48 \mathrm{GPa}$ & 0.367 & $11.17 \mathrm{GPa}$ \\
$\mathrm{y}(\mathrm{yz})$ & $16.85 \mathrm{GPa}$ & 0.177 & $9.35 \mathrm{GPa}$ \\
$\mathrm{z}(\mathrm{xz})$ & $59.14 \mathrm{GPa}$ & 0.278 & $24.38 \mathrm{GPa}$ \\
\hline \hline
\end{tabular}

\section{Electromagnetic-Structural COUPLing ANALYsis} OF MJ TOROIDAL SMES MAGNET

\section{A. Electromagnetic Analysis}

The toroidal SMES magnet is cooled by forced flow cooling method (liquid helium) and the operation temperature is $30 \mathrm{~K}$. The maximum operating current, the maximum magnetic field, the inductance and the storage energy are $3.3 \mathrm{kA}, 3.42$ $\mathrm{T}, 0.57 \mathrm{H}$, and $3.1 \mathrm{MJ}$, respectively. The main design parameters of a $3 \mathrm{MJ}$ toroidal SMES magnet are summarized in TABLE III.

TABLE III

THE MAIN DESIGN PARAMETERS OF A 3 MJ TOROIDAL SMES MAGNET

\begin{tabular}{ll}
\hline \hline Item & Value \\
\hline Number of element coil & 16 \\
Inductance of toroidal magnet/H & 0.57 \\
The maximum value of magnetic field/T & 3.42 \\
The maximum value of operating current $/ \mathrm{kA}$ & 3.3 \\
Operating temperature/K & 30 \\
Storage energy/MJ & 3.1 \\
Total length of HTS wire/km & 25.6 \\
\hline \hline
\end{tabular}

The magnetic field pattern of an element coil of a $3 \mathrm{MJ}$ toroidal SMES magnet is shown in Fig. 5. It can be seen that the magnetic field is confined to the inner diameter and near the center of the toroidal SMES magnet. Those positions may be the danger zone in which the toroidal SMES magnet can be easily damaged.

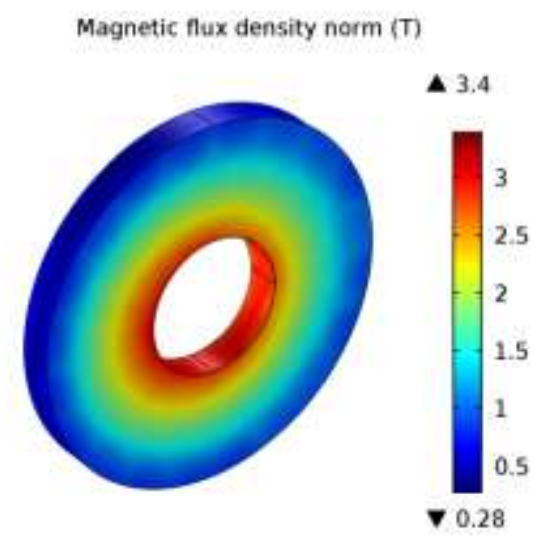

Fig. 5. Magnetic field pattern of an element coil of a 3 MJ toroidal SMES magnet

\section{B. Mechanical Analysis}

The mechanical stresses are caused by the Lorentz force. When the stress on the HTS wires exceeds a critical value, the current carrying capacity of HTS wires will be damaged. Even a fracture of HTS wires and the deformation of magnet coils will occur. Consequently, an analysis of the mechanical properties of an MJ-class toroidal SMES magnet wound by a composite HTS conductor is necessary in order to ensure its stability during operation [7].

For the SMES magnet with nonmagnetic material, the balanced relationship between the Lorentz force and the stress is described in the following equations [8].

$$
\begin{aligned}
& \nabla \times B=\mu_{0} J \quad \nabla \cdot B=0 \\
& J \times B+\Delta \cdot S=0
\end{aligned}
$$

Where, $J$ is the current density, $B$ is the magnetic density and $S$ is the stress tensor. The magnetic density can be examined by (4). Besides, the stress is caused by the Lorenz force generated by interaction between operating current and magnetic field from (5).

The $\mathrm{X}, \mathrm{Y}$ and $\mathrm{Z}$ components of the Lorentz force of an element coil are calculated in this paper. And $\mathrm{X}, \mathrm{Y}$ and $\mathrm{Z}$ components of the Lorentz force are $-0.128 \mathrm{MN}, 0.373 \times 10^{-3}$ $\mathrm{MN}$, and $0.362 \times 10^{-3} \mathrm{MN}$ respectively. Therefore, $\mathrm{Y}$ and $\mathrm{Z}$ components of the Lorentz force are very small and the Lorentz force is gathered in the central direction of a toroidal SMES magnet.

\section{Electromagnetic-Structural Coupling Analysis and Results}

The sequential coupling analysis method is used in this paper. More specifically, the results of a magnetic field analysis will be used as the load for solid mechanics analysis sequentially. The flow chart in Fig. 6 shows the electromagnetic-structural coupling calculation of the toroidal SMES magnet. 


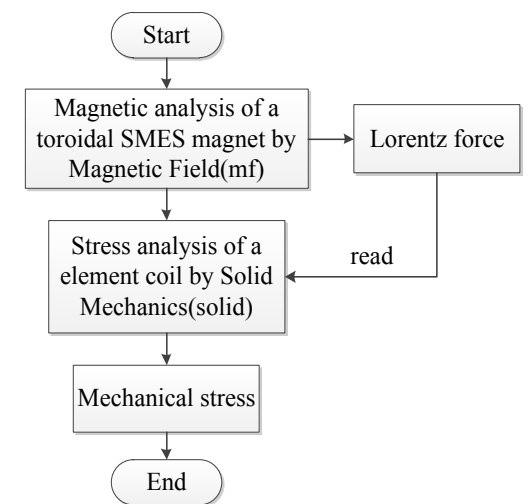

Fig. 6. Electromagnetic-structural coupling calculation of the toroidal SMES magnet

Fig. 7 shows the configuration of a magnet coil and its structural support parts of a $3 \mathrm{MJ}$ toroidal SMES magnet used for electromagnetic-structural coupling calculation.

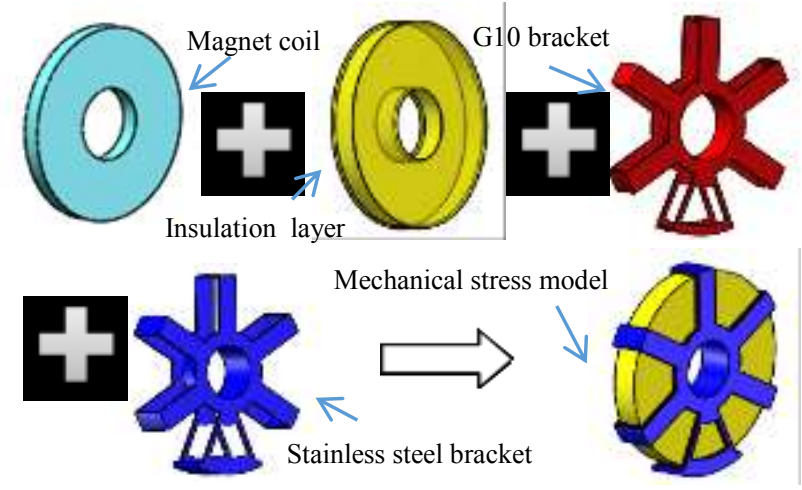

Fig. 7. Configuration of magnet coil and its structural support parts

Based on electromagnetic-structural coupling calculation, the radial stress $\sigma_{r}$ and the hoop stress $\sigma_{h}$ of a toroidal SMES magnet can be calculated with the following formulas (6) and (7) [9].

$$
\begin{aligned}
& \sigma_{r}=0.5\left(\sigma_{x}+\sigma_{z}\right)+0.5\left(\sigma_{x}-\sigma_{z}\right) \cos 2 \theta+\tau_{x z} \sin 2 \theta \\
& \sigma_{h}=0.5\left(\sigma_{x}+\sigma_{z}\right)-0.5\left(\sigma_{x}-\sigma_{z}\right) \cos 2 \theta-\tau_{x z} \sin 2 \theta
\end{aligned}
$$

Fig. 8 shows the Von-Mises stress, the radial stress, and the hoop stress of a magnet coil respectively. The maximum VonMises stress of a magnet coil is $138 \mathrm{MPa}$ as shown in Fig. 8 (a), the maximum radial stress of a magnet coil is $27.4 \mathrm{MPa}$ as shown in Fig. 8 (b) and the maximum hoop stress of a magnet coil is $14.4 \mathrm{MPa}$ as shown in Fig. 8 (c). All of the above stress results are much lower than the maximum yield stress (150 $\mathrm{MPa}$ ) of the REBCO coated conductor.

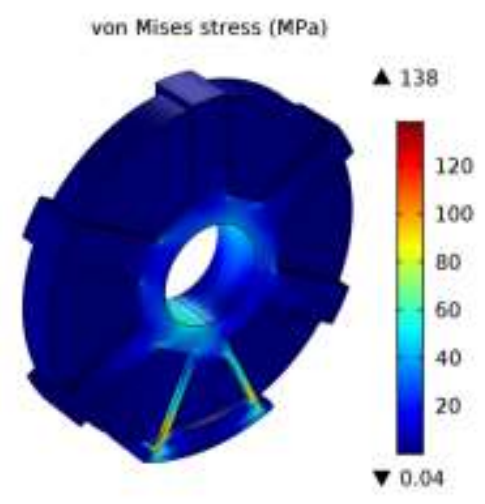

(a) Von-Mises stress of the magnet coil Radial stress of a element coil(mpa)

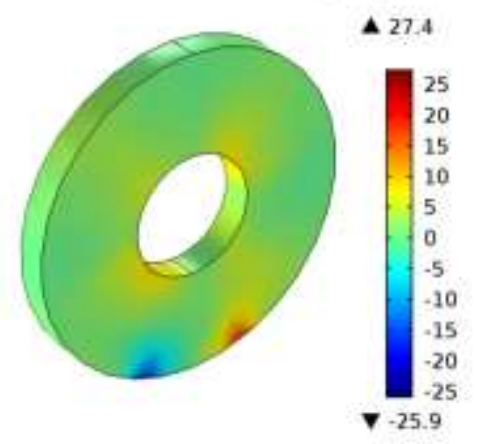

(b) Radial stress of the magnet coil Hoop stress of a element coil(MPa)

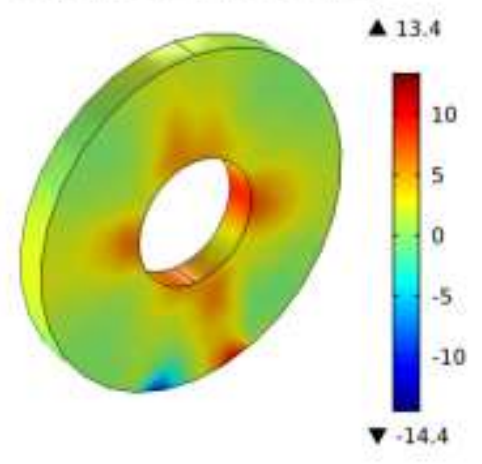

(c) Hoop stress of the magnet coil

Fig. 8. Electromagnetic-structural stress analysis coupling results According to the above analysis, the mechanical stresses of a magnet coil wound by a composite HTS conductor are perfectly acceptable. The stress imposed on the HTS wire does not exceed the maximum rated tensile stress, so it will not cause critical current degradation and the $3 \mathrm{MJ}$ toroidal SMES magnet will be safe. Besides, the mechanical stress results can be used for the optimum structural design of composite HTS conductors, which will be discussed in future papers.

\section{CONCLUSION}

Using the equivalent material properties method, a simplified model of composite HTS conductors is built to calculate their equivalent orthotropic material properties. By means of the sequential coupling analysis method, an electromagnetic-structural coupling calculation is achieved. With an analysis of the mechanical properties of a $3 \mathrm{MJ}$ toroidal SMES magnet wound by composite HTS conductors, this paper finds that the maximum hoop stress of an SMES coil is lower than the maximum yield stress of an REBCO coated conductor. Therefore, an REBCO coated conductor will not be subject to critical current degradation while the reliable and stable operation of the $3 \mathrm{MJ}$ toroidal SMES magnet can be ensured. Such fundamental data will prove useful in designing MJ-class toroidal SMES magnets wound by composite HTS conductors. 


\section{REFERENCES}

[1] Jiahui Zhu, Weijia Yuan, Ming Qiu et al., "Experimental demonstration and application planning of high temperature superconducting energy storage system for renewable power grids," Applied Energy, 2015, 137(1):692-698.

[2] Mohd. Hasan Ali, Bin Wu, Roger A. Dougal, "An overview of SMES applications in power and energy systems," IEEE Transactions on Sustainable Energy. 2010, 1(1):38-47.

[3] C. Barth et al., "Temperature-and field dependent characterization of a twisted stacked-tape cable, Supercond. Sci. Technol., vol. 28, no. 4, Feb. 2015, Art. ID 045015.

[4] M. Takayasu, L. Chiesa, N. C. Allen, and J. V. Minervini, "Present status and recent developments of Twisted Stacked-Tape Cable (TSTC) conductor," presented at the HTS4Fusion Conductor Workshop, Pieve Santo Stefano, Italy, Sep. 11-12, 2015.

[5] M. Takayasu, L. Chiesa, and J. V. Minervini, "Investigation of REBCO twisted stacked-tape cable conductor performance," J. Phys. Conf. Ser., vol. 507, no. 2, 2014, Art. ID 022040.

[6] J.-L. Batoz and G. Dhatt, Modélisation des structures par éléments finis. Paris, France: Solides Élastiques, 1990.

[7] K. Shimada, S. Hanai, L. Kushida, K. Hirabayashi, T. Kobayashi, T. Simonosono, H. Hayashi, K. Tsutsumi, F. Irie, Y. Horiuchi, and T. Ezaki, "Experimental and analytical studies on mechanical behavior of superconducting coil for SMES," IEEE Trans. Appl. Supercond., vol. 11, no. 1, pp. 1912-1915, 2001.

[8] M.-J. Park, S.-Y. Kwak, W.-S. Kim, S.-W. Lee, S.-Y. Hahn, J.-K.Lee, J.-H. Han, K.-D. Choi, H.-K. Jung, K.-C. Seong, and S.-Y. Hahn, "Stress analysis of HTS magnet for a $600 \mathrm{~kJ}$ SMES," IEEE Trans. Appl. Supercond., vol. 17, no. 2, pp. 1994-1997, 2007.

[9] Kwangmin Kim, Sangjin Lee, Seokho Kim, Minwon Park, In-Keun Yu, Ji-Kwang Lee, Se-Yeon Lee, Kyeongdal Choi, Ki-Cheol Seong, and Hankil Yeom, "Stress analysis for toroid-type HTS SMES coil and bobbin structure considering large parallel magnetic field," IEEE Trans. Appl. Supercond., vol. 21, no. 3, pp. 2275-2278, 2011. 